\title{
STABLE AND UNSTABLE BIFURCATION IN THE VON KÁRMÁN PROBLEM FOR A CIRCULAR PLATE
}

\author{
ANDREI BORISOVICH AND JOANNA JANCZEWSKA
}

Received 20 February 2004

In this work, we study bifurcation in the von Kármán equations for a thin circular elastic plate which lies on the elastic base and is simply supported and subjected to a compressive force along the boundary. Applying analytical methods, we prove the existence of stable and unstable simple bifurcation points in the solution set of these equations.

\section{Introduction}

In this paper, we study a certain problem of partial differential equations which derives from the mechanics of elastic constructions. Our goal is to find forms of equilibrium of a thin circular elastic plate on the elastic foundation under the action of a compressive force. A form of equilibrium of the plate is a pair of functions $(w, \sigma)$ composed of a deflection function $w$ and a stress function $\sigma$.

Let $D$ be the unit disk in $R^{2}$ centered at the origin ( $D$ corresponds with the shape of the plate) and let $\mu \in(0,1)$. Forms of equilibrium of the plate may be found as solutions $(w, \sigma) \in C^{4, \mu}(\bar{D}) \times C^{4, \mu}(\bar{D})$ of the following differential equations:

$$
\begin{gathered}
\Delta^{2} w-[w, \sigma]-\alpha w_{u u}\left(3 w_{u}^{2}+w_{v}^{2}\right)-\alpha w_{v v}\left(3 w_{v}^{2}+w_{u}^{2}\right) \\
-4 \alpha w_{u v} w_{u} w_{v}+2 \alpha \Delta w+\beta w-\gamma w^{3}=0, \\
\Delta^{2} \sigma+\frac{1}{2}[w, w]=0, \quad(u, v) \in D, \\
w=\Delta w=0, \\
\sigma=\Delta \sigma=0, \quad(u, v) \in \partial D
\end{gathered}
$$

with two positive parameters $\alpha, \beta$ and a positive constant $\gamma$. The parameter $\alpha$ is a value of the loading (the compressive force on the length unit of $\partial D$ ), $\beta$ is a parameter of the elastic foundation (it corresponds with the force produced by the foundation), and $\gamma$ is a constant that characterizes the foundation. The operator $[\cdot, \cdot]$ is given as follows:

$$
[w, \sigma]=w_{u u} \sigma_{v v}-2 w_{u v} \sigma_{u v}+w_{v v} \sigma_{u u}
$$

and $\Delta$ denotes the Laplace operator. 
Our partial differential equations are called the von Kármán equations. The first equations that described the buckling of a thin elastic plate were proposed by Theodore von Kármán in 1910. He studied two nonlinear partial differential equations with Dirichlet boundary conditions and one parameter corresponding to a compressive force. Many people have studied his equations with extra assumptions on the shape of the plate and with different boundary conditions (clamped, simply supported, free) by the use of numerical, analytical and variational methods. Among the previous studies of the von Kármán equations are those of $[1,2,5,6,9,11]$. The equations with two parameters appeared for the first time in [10], and later in [4, 7]. The equations (1.1) describe the phenomenon of the buckling of the plate only approximately. They were derived from the rule of conservation of energy. Writing the formula of energy of the plate we took into account the terms up till the 4 th order.

We note that $(0,0) \in C^{4, \mu}(\bar{D}) \times C^{4, \mu}(\bar{D})$ is a solution of (1.1) for every $\alpha$ and $\beta$. This is of course the solution corresponding with the undeflected position of the plate. Experiments show that for some values of $\alpha$ and $\beta$ the plate may deflect out, that is, equilibrium forms other than $(0,0)$ are possible. We prove this by the use of bifurcation theory.

From now on, a solution of $(1.1)$ is called a 4-tuple $(w, \sigma, \alpha, \beta)$ such that:

(i) $(w, \sigma) \in C^{4, \mu}(\bar{D}) \times C^{4, \mu}(\bar{D})$,

(ii) $(\alpha, \beta) \in R_{+}^{2}$,

(iii) $(w, \sigma)$ satisfies pointwise (1.1) with the parameters $\alpha, \beta$.

Let $\Gamma=\left\{(0,0, \alpha, \beta) \in C^{4, \mu}(\bar{D}) \times C^{4, \mu}(\bar{D}) \times R_{+}^{2}: \alpha, \beta \in R_{+}\right\}$. Every point from $\Gamma$ is called a trivial solution of (1.1). Every solution of (1.1) that does not belong to $\Gamma$ is called $a$ nontrivial solution. A trivial solution of (1.1) that belongs to the closure of the set of nontrivial solutions is a bifurcation point of (1.1).

Our main purpose is the study of the existence of bifurcation points of (1.1). In Section 2, we write our equations in the form of an operator equation $F(w, \sigma, \alpha, \beta)=0$ and we collect basic properties of $F$. In Section 3, we review some of the standard notions and facts of bifurcation theory. Section 4 is devoted to the study of stable and unstable bifurcation in the von Kármán problem.

\section{Properties of the von Kármán equations}

In this section, we transform the von Kármán equations into the operator equation $F(w, \sigma, \alpha, \beta)=0$ in Banach spaces. We discuss a few important properties of $F$ that we apply to the study of bifurcation in the next section.

Let $C_{0,0}^{4, \mu}(\bar{D})=\left\{f \in C^{4, \mu}(\bar{D}): f_{\mid \partial D}=\Delta f_{\mid \partial D}=0\right\}$. Set $X=C_{0,0}^{4, \mu}(\bar{D}) \times C_{0,0}^{4, \mu}(\bar{D})$ and $Y=$ $C^{0, \mu}(\bar{D}) \times C^{0, \mu}(\bar{D})$. The Hölder spaces are considered here with their standard and wellknown norms. The norms in $X$ and $Y$ are defined as the maximum of the norms of both coordinates. Let $F_{1}, F_{2}: X \times R_{+}^{2} \rightarrow C^{0, \mu}(\bar{D})$ be given by

$$
\begin{aligned}
F_{1}(x, p)= & \Delta^{2} w-[w, \sigma]+2 \alpha \Delta w-\alpha w_{u u}\left(3 w_{u}^{2}+w_{v}^{2}\right) \\
& -\alpha w_{v v}\left(3 w_{v}^{2}+w_{u}^{2}\right)-4 \alpha w_{u v} w_{u} w_{v}+\beta w-\gamma w^{3}, \\
F_{2}(x, p)= & -\Delta^{2} \sigma-\frac{1}{2}[w, w]
\end{aligned}
$$


where $x=(w, \sigma)$ and $p=(\alpha, \beta)$. The map $F: X \times R_{+}^{2} \rightarrow Y$ is defined by

$$
F=\left(F_{1}, F_{2}\right) .
$$

We see at once that the von Kármán equations are equivalent to the operator equation

$$
F(x, p)=0
$$

$F$ is easily seen to be $C^{\infty}$-smooth, and a trivial verification shows that for every $p \in R_{+}^{2}$ and $h \in X$

$$
F_{x}^{\prime}(0, p) h=\left(\Delta^{2} z+2 \alpha \Delta z+\beta z,-\Delta^{2} \eta\right)
$$

where $h=(z, \eta)$. We collect now several important facts concerning $F_{x}^{\prime}(0, p): X \rightarrow Y$. All the details can be found in $[7,8]$.

Fact 2.1. $F_{x}^{\prime}(0, p): X \rightarrow Y$ is a Fredholm map of index zero for every $p \in R_{+}^{2}$.

The proof of this fact is immediate. It is based on the observation that

$$
F_{x}^{\prime}(0, p) h=A(h)+B(h)
$$

where $A(h)=\left(\Delta^{2} z,-\Delta^{2} \eta\right)$ is an isomorphism and $B(h)=(2 \alpha \Delta z+\beta z, 0)$ is completly continuous.

Set $C_{0}^{2, \mu}(\bar{D})=\left\{f \in C^{2, \mu}(\bar{D}): f_{\mid \partial D}=0\right\}$. To simplify notation, we use the same letter I for the natural embedding of $C_{0}^{2, \mu}(\bar{D})$ into $C^{0, \mu}(\bar{D})$ and for the natural embedding of $C_{0,0}^{4, \mu}(\bar{D})$ into $C^{0, \mu}(\bar{D})$.

Fix $p=(\alpha, \beta) \in R_{+}^{2}$. Let $\delta=\alpha^{2}-\beta$. Set $a=-\alpha-\sqrt{\delta}$ and $b=-\alpha+\sqrt{\delta}$, provided $\delta \geq 0$. Let $\Delta-a I, \Delta-b I: C_{0}^{2, \mu}(\bar{D}) \rightarrow C^{0, \mu}(\bar{D})$. We will describe the kernel of $F_{x}^{\prime}(0, p)$, denoted by $N(p)$. Since $\Delta^{2}: C_{0,0}^{4, \mu}(\bar{D}) \rightarrow C^{0, \mu}(\bar{D})$ is an isomorphism, it suffices to research the kernel of $\Delta^{2}+2 \alpha \Delta+\beta I: C_{0,0}^{4, \mu}(\bar{D}) \rightarrow C^{0, \mu}(\bar{D})$.

Fact 2.2. (i) If $\delta<0$ then $\operatorname{ker}\left(\Delta^{2}+2 \alpha \Delta+\beta I\right)=\{0\}$.

(ii) If $\delta=0$ then $\operatorname{ker}\left(\Delta^{2}+2 \alpha \Delta+\beta I\right)=\operatorname{ker}(\Delta-a I)$.

(iii) If $\delta>0$ then $\operatorname{ker}\left(\Delta^{2}+2 \alpha \Delta+\beta I\right)=\operatorname{ker}(\Delta-a I) \oplus \operatorname{ker}(\Delta-b I)$.

It is well-known that $\lambda<0$ is an eigenvalue of $\Delta: C_{0}^{2, \mu}(\bar{D}) \rightarrow C^{0, \mu}(\bar{D})$ if and only if there is $k \in N \cup\{0\}$ such that $\sqrt{-\lambda}$ is a root of the Bessel function $J_{k}: R \rightarrow R$ given by

$$
J_{k}(s)=\frac{1}{\pi} \int_{0}^{\pi} \cos (s \sin t-k t) d t
$$

Furthermore, if $J_{k}(\sqrt{-\lambda})=0$ for a certain $k \in N$ then $\operatorname{dim} \operatorname{ker}(\Delta-\lambda I)=2$ and $\operatorname{ker}(\Delta-$ $\lambda I)=\operatorname{span}\left\{J_{k}(\sqrt{-\lambda} r) \cos (k \varphi), J_{k}(\sqrt{-\lambda} r) \sin (k \varphi)\right\}$, where $(r, \varphi)$ are polar coordinates. If $J_{0}(\sqrt{-\lambda})=0$ then $\operatorname{ker}(\Delta-\lambda I)=\operatorname{span}\left\{J_{0}(\sqrt{-\lambda} r)\right\}$. Combining this with Fact 2.2 we conclude that the dimension $N(p)$ is no greater than 4 . We are able to determine when exactly $N(p)$ is one, two, three, or four dimensional. In this paper, we are interested in a one dimensional case. 
Fact 2.3. $N(p)$ is one dimensional if and only if one of the following conditions holds:

(i) $\delta=0$ and $J_{0}(\sqrt{-a})=0$,

(ii) $\delta>0, J_{0}(\sqrt{-a})=0$ and $J_{k}(\sqrt{-b}) \neq 0$ for every $k \in N \cup\{0\}$,

(iii) $\delta>0, J_{0}(\sqrt{-b})=0$ and $J_{k}(\sqrt{-a}) \neq 0$ for every $k \in N \cup\{0\}$.

Let $\langle\cdot, \cdot\rangle: Y \times Y \rightarrow R$ be given by

$$
\langle(w, \sigma),(z, \eta)\rangle=\frac{1}{\pi} \iint_{D}(w z+\sigma \eta) d u d v .
$$

Formula (2.7) determines the scalar product both in $Y$ and $X$. Since the convergence in Hölder spaces implies the uniform convergence, this scalar product is continuous in $X$ and $Y$. Define $E: X \times R_{+}^{2} \rightarrow R$ as follows

$$
\begin{aligned}
E(x, p)= & \frac{1}{2 \pi} \iint_{D}\left((\Delta w)^{2}-(\Delta \sigma)^{2}-[w, w] \sigma\right) d u d v \\
& +\frac{1}{2 \pi} \iint_{D}\left(-2 \alpha\left(w_{u}^{2}+w_{v}^{2}\right)+\frac{\alpha}{2}\left(w_{u}^{2}+w_{v}^{2}\right)^{2}\right) d u d v \\
& +\frac{1}{2 \pi} \iint_{D}\left(\beta w^{2}-\frac{1}{2} \gamma w^{4}\right) d u d v
\end{aligned}
$$

Note that $E$ is $C^{\infty}$-smooth.

Fact 2.4. $F$ is a variational gradient of $E$ with respect to the scalar product (2.7), that is, $E_{x}^{\prime}(x, p) h=\langle F(x, p), h\rangle$ for all $x, h \in X$ and $p \in R_{+}^{2}$.

Proof. Take $x=(w, \sigma), h=(z, \eta) \in X$ and $p \in R_{+}^{2}$. Since $E$ is $C^{\infty}$-smooth, the Fréchet derivative of $E$ is equal to the Gateux derivative of this functional. An easy computation shows that

$$
\begin{aligned}
\frac{d}{d t} E(x+t h)_{\mid t=0}= & \frac{1}{\pi} \iint_{D} \Delta w \Delta z d u d v-\frac{1}{\pi} \iint_{D} \Delta \sigma \Delta \eta d u d v \\
& -\frac{1}{\pi} \iint_{D} \sigma[w, z] d u d v-\frac{1}{\pi} \iint_{D} \frac{1}{2}[w, w] \eta d u d v \\
& -\frac{1}{\pi} \iint_{D} 2 \alpha\left(w_{u} z_{u}+w_{v} z_{v}\right) d u d v \\
& +\frac{1}{\pi} \iint_{D} \alpha\left(w_{u}^{2}+w_{v}^{2}\right)\left(w_{u} z_{u}+w_{v} z_{v}\right) d u d v \\
& +\frac{1}{\pi} \iint_{D}\left(\beta w z-\gamma w^{3} z\right) d u d v .
\end{aligned}
$$

The operator $\Delta: C_{0}^{2, \mu}(\bar{D}) \rightarrow C^{0, \mu}(\bar{D})$ is symmetric with respect to the scalar product in $L^{2}(D)$. Hence

$$
\begin{aligned}
& \iint_{D} \Delta w \Delta z d u d v=\iint_{D}\left(\Delta^{2} w\right) z d u d v \\
& \iint_{D} \Delta \sigma \Delta \eta d u d v=\iint_{D}\left(\Delta^{2} \sigma\right) \eta d u d v
\end{aligned}
$$


Applying the Fubini theorem and the method of integrating by parts we receive

$$
\begin{aligned}
& \iint_{D} \sigmaw, z] d u d v \\
&= \int_{-1}^{1}\left(\int_{-\sqrt{1-u^{2}}}^{\sqrt{1-u^{2}}} \sigma w_{u u} z_{v v} d v\right) d u+\int_{-1}^{1}\left(\int_{-\sqrt{1-v^{2}}}^{\sqrt{1-v^{2}}} \sigma w_{v v} z_{u u} d u\right) d v \\
&-2 \int_{-1}^{1}\left(\int_{-\sqrt{1-v^{2}}}^{\sqrt{1-v^{2}}} \sigma w_{u v} z_{u v} d u\right) d v \\
&= \iint_{D}[w, \sigma] z d u d v, \\
& \iint_{D}\left(w_{u} z_{u}+w_{v} z_{v}\right) d u d v \int_{-1}^{1}\left(\int_{-\sqrt{1-v^{2}}}^{\sqrt{1-v^{2}}} w_{u} z_{u} d u\right) d v+\int_{-1}^{1}\left(\int_{-\sqrt{1-u^{2}}}^{\sqrt{1-u^{2}}} w_{v} z_{v} d v\right) d u \\
&=-\iint_{D}\left(\Delta w^{2} z d u d v,\right. \\
& \iint_{D}\left(w_{u}^{2}+w_{v}^{2}\right)\left(w_{u} z_{u}+w_{v} z_{v}\right) d u d v \\
&=\int_{-1}^{1}\left(\int_{-\sqrt{1-v^{2}}}^{\sqrt{1-v^{2}}}\left(w_{u}^{2}+w_{v}^{2}\right) w_{u} z_{u} d u\right) d v \\
& \quad+\int_{-1}^{1}\left(\int_{-\sqrt{1-u^{2}}}^{\sqrt{1-u^{2}}}\left(w_{u}^{2}+w_{v}^{2}\right) w_{v} z_{v} d v\right) d u \\
&=-\iint_{D} w_{u u}\left(3 w_{u}^{2}+w_{v}^{2}\right) z d u d v-\iint_{D} w_{v v}\left(3 w_{v}^{2}+w_{u}^{2}\right) z d u d v \\
& \quad-\iint_{D} 4 w_{u} w_{v} w_{u v} z d u d v .
\end{aligned}
$$

Consequently,

$$
\begin{aligned}
E_{x}^{\prime}(x, p) h= & \frac{1}{\pi} \iint_{D}\left(\Delta^{2} w-[w, \sigma]+2 \alpha \Delta w-\alpha w_{u u}\left(3 w_{u}^{2}+w_{v}^{2}\right)\right) z d u d v \\
& +\frac{1}{\pi} \iint_{D}\left(-\alpha w_{v v}\left(3 w_{v}^{2}+w_{u}^{2}\right)-4 \alpha w_{u} w_{v} w_{u v}\right) z d u d v \\
& +\frac{1}{\pi} \iint_{D}\left(\beta w-\gamma w^{3}\right) z d u d v+\frac{1}{\pi} \iint_{D}\left(-\Delta^{2} \sigma-\frac{1}{2}[w, w]\right) \eta d u d v \\
= & \frac{1}{\pi} \iint_{D}\left(F_{1}(x, p) z+F_{2}(x, p) \eta\right) d u d v \\
= & \langle F(x, p), h\rangle .
\end{aligned}
$$

\section{Introduction to bifurcation theory}

We recall a few notions and facts of bifurcation theory. To shorten notation, we use the same letters as in the von Kármán problem. The following assumptions will be needed 
throughout this section:

(i) $X$ and $Y$ are Banach spaces,

(ii) $F$ is a $C^{1}$-smooth map defined in a certain neighbourhood of a point $\left(0, \alpha_{0}\right) \in$ $X \times R^{n}$ to $Y$,

(iii) $F(0, \alpha)=0$ for every $\alpha$ in a certain neighbourhood of $\alpha_{0}$.

Consider the equation

$$
F(x, \alpha)=0
$$

The set $\Gamma=\left\{(0, \alpha) \in X \times R^{n}: F(0, \alpha)=0\right\}$ is composed of trivial solutions of (3.1). Each point $(x, \alpha) \in X \times R^{n}$ such that $F(x, \alpha)=0$ and $x \neq 0$ is a nontrivial solution of (3.1).

Definition 3.1. $\left(0, \alpha_{0}\right) \in \Gamma$ is a bifurcation point of (3.1) with respect to the set $\Gamma$ if it belongs to the closure of the set of nontrivial solutions of (3.1).

Let $N\left(\alpha_{0}\right)=\operatorname{ker} F_{x}^{\prime}\left(0, \alpha_{0}\right)$. If $F_{x}^{\prime}\left(0, \alpha_{0}\right): X \rightarrow Y$ is a Fredholm operator of index 0 then the necessary condition for bifurcation at $\left(0, \alpha_{0}\right)$ is that $\operatorname{dim} N\left(\alpha_{0}\right)>0$. It is a simple consequence of the theorem on the implicit function. If $\left(0, \alpha_{0}\right)$ is a bifurcation point such that $N\left(\alpha_{0}\right)$ is one dimensional, then it is called a simple bifurcation point. Otherwise, it is a multiple bifurcation point.

In this paper, we are interested in simple bifurcation points. One of the basic theorems on simple bifurcation points was proved by Crandall and Rabinowitz in 1971. Below we formulate this theorem in the form adopted to our needs.

Theorem 3.2. Assume that $F: X \times R^{1} \rightarrow Y$ satisfies the following conditions:

(1) $F$ is a $C^{r}$-map, $r \geq 3$,

(2) $\operatorname{dim} N\left(\alpha_{0}\right)=1, F_{x}^{\prime}\left(0, \alpha_{0}\right) e=0, e \neq 0$,

(3) $\operatorname{codim} I m F_{x}^{\prime}\left(0, \alpha_{0}\right)=1$,

(4) $F_{\alpha \alpha}^{\prime \prime}\left(0, \alpha_{0}\right) \in \operatorname{Im} F_{x}^{\prime}\left(0, \alpha_{0}\right)$,

(5) $F_{x \alpha}^{\prime \prime}\left(0, \alpha_{0}\right) \notin \operatorname{Im} F_{x}^{\prime}\left(0, \alpha_{0}\right)$.

Under the above assumptions, the solution set of (3.1) in a certain neighbourhood of $\left(0, \alpha_{0}\right)$ is composed of $\Gamma$ and $a C^{r-2}$-smooth curve $\Lambda$. $\Gamma$ and $\Lambda$ intersect at $\left(0, \alpha_{0}\right)$ only, and $\Lambda$ is parametrized as follows

$$
\Lambda=\{(\hat{x}(t), \hat{\alpha}(t)):|t|<\varepsilon\},
$$

where $\hat{x}(0)=0, \hat{x}^{\prime}(0)=e$, and $\hat{\alpha}(0)=\alpha_{0}$.

Suppose that (3.1) describes a certain problem coming from the mechanics of elastic plates and a parameter $\alpha \in R$ denotes the loading. We may distinguish stable and unstable bifurcation points. If the shape of the plate changes continuously by crossing $\alpha_{0}$, then $\left(0, \alpha_{0}\right)$ is called a stable bifurcation point. Otherwise, it is called an unstable bifurcation point. We present now a simple way of the study of stable and unstable bifurcation points.

Let $X$ be a linear subspace of $Y$. Assume that there is given a scalar product in $Y$, $\langle\cdot, \cdot\rangle: Y \times Y \rightarrow R$, that is continuous in $X$ and $Y$. Let $F$ satisfy the following conditions:

$\left(\mathrm{H}_{1}\right) F$ is a $C^{r}$-map, $r \geq 3$,

$\left(\mathrm{H}_{2}\right) \operatorname{dim} N\left(\alpha_{0}\right)=1, F_{x}^{\prime}\left(0, \alpha_{0}\right) e=0,\langle e, e\rangle \neq 0$,

$\left(\mathrm{H}_{3}\right) F_{x}^{\prime}\left(0, \alpha_{0}\right): X \rightarrow Y$ is a Fredholm map of index 0 , 


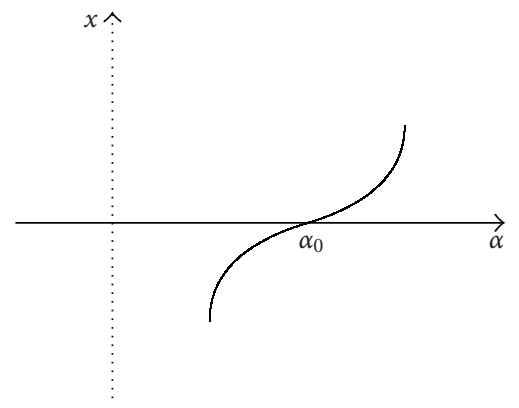

Figure 3.1. Transcritical bifurcation.

$\left(\mathrm{H}_{4}\right) F$ is a variational gradient of a certain $C^{r+1}$-smooth functional $E$ with respect to the scalar product in $Y$, that is, $E_{x}^{\prime}(x, \alpha) h=\langle F(x, \alpha), h\rangle$ for every $(x, \alpha)$ in the domain of $F$ and $h \in X$,

$\left(\mathrm{H}_{5}\right) E_{x x \alpha}^{\prime \prime \prime}\left(0, \alpha_{0}\right) \neq 0$.

It is easy to check that $F$ satisfies the assumptions of Theorem 3.2. Applying the scheme of the finite-dimensional reduction by Lyapunov and Schmidt we reduce the problem of existence of bifurcation from the trivial solution of (3.1) to the bifurcation problem for a certain equation in $R$ :

$$
\Phi_{\xi}^{\prime}(\xi, \alpha)=0
$$

where $\xi \in R$. There are different methods of finding $\Phi$. We use the method described by Sapronov. To this aim consider the equation

$$
F(x, \alpha)+(\xi-\langle x, e\rangle) e=0 .
$$

By the theorem on the implicit function, there exist two open sets $U \subset X$ and $V \subset R^{2}$ such that $0 \in U$ and $\left(0, \alpha_{0}\right) \in V$ and there exists a $C^{r}$-map $\tilde{x}: V \rightarrow U$ such that $\tilde{x}\left(0, \alpha_{0}\right)=0$ and the solution set of (3.4) in $U \times V$ is the graph of $\tilde{x}$. Then $\Phi: V \rightarrow R$ is given by

$$
\Phi(\xi, \alpha)=-E(\tilde{x}(\xi, \alpha), \alpha)+\frac{1}{2}(\xi-\langle\tilde{x}(\xi, \alpha), e\rangle)^{2} .
$$

$\Phi$ is called the key function.

Theorem 3.3 (Borysowicz, see [3, 4]). Under the above assumptions, the solution set of (3.1) in a certain neighbourhood of $\left(0, \alpha_{0}\right)$ is composed of $\Gamma$ and a $C^{r-2}$-smooth curve $\Lambda$. The curves $\Gamma$ and $\Lambda$ intersect at $\left(0, \alpha_{0}\right)$ only.

(i) If $\Phi_{\xi \xi \alpha}^{\prime \prime \prime}\left(0, \alpha_{0}\right) \neq 0$ and $\Phi_{\xi \xi \xi}^{\prime \prime \prime}\left(0, \alpha_{0}\right) \neq 0$ then $\left(0, \alpha_{0}\right)$ is a transcritical bifurcation point and $\Lambda$ is parametrized as follows:

$$
\Lambda: \hat{x}(\alpha)=C\left(\alpha-\alpha_{0}\right) e+o\left(\left|\alpha-\alpha_{0}\right|\right), \quad \alpha \in\left(\alpha_{0}-\varepsilon, \alpha_{0}+\varepsilon\right),
$$

where $C=-2 \Phi_{\xi \xi \alpha}^{\prime \prime \prime}\left(0, \alpha_{0}\right) / \Phi_{\xi \xi \xi}^{\prime \prime \prime}\left(0, \alpha_{0}\right)$ and $\varepsilon>0$ is small enough (see Figure 3.1). 
896 Stable and unstable bifurcation in von Kármán problem

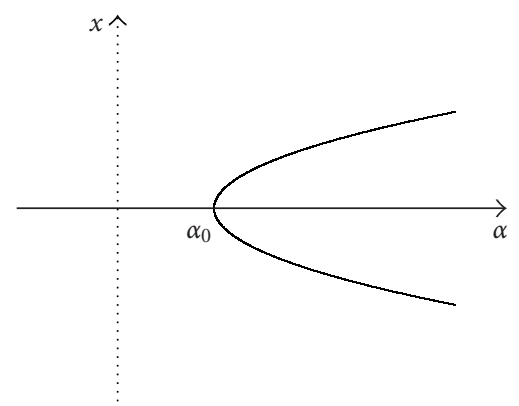

Figure 3.2. Postcritical bifurcation.

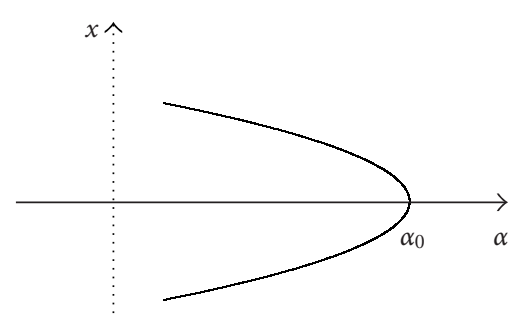

Figure 3.3. Subcritical bifurcation.

(ii) If $\Phi_{\xi \xi \alpha}^{\prime \prime \prime}\left(0, \alpha_{0}\right) \neq 0, \Phi_{\xi \xi \xi}^{\prime \prime \prime}\left(0, \alpha_{0}\right)=0$ and $\Phi_{\xi \xi \xi \xi}^{(4)}\left(0, \alpha_{0}\right) \neq 0$ then the phenomenon of $b i$ furcation at $\left(0, \alpha_{0}\right)$ depends on

$$
D=\frac{-6 \Phi_{\xi \xi \alpha}^{\prime \prime \prime}\left(0, \alpha_{0}\right)}{\Phi_{\xi \xi \xi \xi}^{(4)}\left(0, \alpha_{0}\right)} .
$$

If $D>0$ then $\left(0, \alpha_{0}\right)$ is a postcritical bifurcation point and $\Lambda$ is parametrized as follows:

$$
\Lambda: \hat{x}(\alpha)= \pm \sqrt{D\left(\alpha-\alpha_{0}\right)} e+o\left(\sqrt{\alpha-\alpha_{0}}\right), \quad \alpha \in\left[\alpha_{0}, \alpha_{0}+\varepsilon\right),
$$

where $\varepsilon>0$ is small enough (see Figure 3.2).

If $D<0$ then $\left(0, \alpha_{0}\right)$ is a subcritical bifurcation point and $\Lambda$ is parametrized as follows:

$$
\Lambda: \hat{x}(\alpha)= \pm \sqrt{|D|\left(\alpha_{0}-\alpha\right)} e+o\left(\sqrt{\alpha_{0}-\alpha}\right), \quad \alpha \in\left(\alpha_{0}-\varepsilon, \alpha_{0}\right]
$$

where $\varepsilon>0$ is small enough (see Figure 3.3).

The proof of this theorem is based on the Crandall-Rabinowitz theorem and the key function method. 


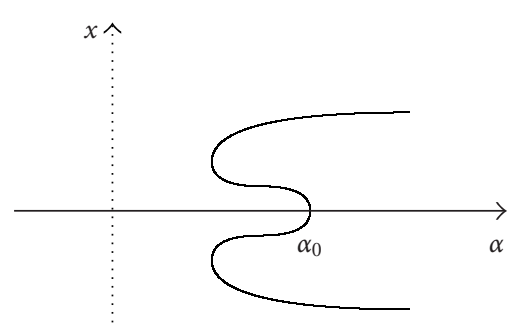

Figure 3.4. Unstable bifurcation.

If (3.1) describes the phenomenon of buckling of an elastic plate then on the strength of experimental results and Theorem 3.3 we conclude that if $D<0$ then $\left(0, \alpha_{0}\right)$ is an unstable bifurcation point. That is we observe bifurcation such as in Figure 3.4.

\section{Stable and unstable bifurcation points in the von Kármán equations}

We come back to the von Kármán problem. Let $p_{0}=\left(\alpha_{0}, \beta_{0}\right) \in R_{+}^{2}$ and $\delta_{0}=\alpha_{0}^{2}-\beta_{0}$. From now on, we assume that $\left(0, p_{0}\right) \in X \times R_{+}^{2}$ is such that $\delta_{0} \geq 0$ and $N\left(p_{0}\right)=1$. Set $a_{0}=$ $-\alpha_{0}-\sqrt{\delta_{0}}$ and $b_{0}=-\alpha_{0}+\sqrt{\delta_{0}}$. By Fact 2.2,

$$
N\left(p_{0}\right)=\operatorname{ker}\left(\Delta-c_{0} I\right) \times\{0\}=\operatorname{span}\left\{\left(J_{0}\left(r \sqrt{-c_{0}}\right), 0\right)\right\},
$$

where

$$
\mathcal{c}_{0}= \begin{cases}a_{0} & \text { if } J_{0}\left(\sqrt{-a_{0}}\right)=0, \\ b_{0} & \text { if } J_{0}\left(\sqrt{-b_{0}}\right)=0 .\end{cases}
$$

Take $e \in N\left(p_{0}\right)$ such that $\langle e, e\rangle=1$. From the above it follows that $e=\left(e_{1}, 0\right), \Delta e_{1}=c_{0} e_{1}$ and there exists $c_{1} \in R \backslash\{0\}$ such that $e_{1}(u, v)=c_{1} J_{0}\left(r \sqrt{-c_{0}}\right)$. Consider the equation

$$
F\left(x, \alpha, \beta_{0}\right)=0
$$

with $F$ defined by (2.2). Let

$$
\gamma\left(p_{0}\right)=\frac{\alpha c_{0}^{2} \int_{0}^{1} r\left(J_{0}^{\prime}\left(r \sqrt{-c_{0}}\right)\right)^{4} d r-2 c_{0}^{3} \int_{0}^{1} r\left(\Delta^{-1}\left((1 / r) J_{0}^{\prime \prime}\left(r \sqrt{-c_{0}}\right) J_{0}^{\prime}\left(r \sqrt{-c_{0}}\right)\right)\right)^{2} d r}{\int_{0}^{1} r J_{0}^{4}\left(r \sqrt{-c_{0}}\right) d r} .
$$

Theorem 4.1. If $\gamma>\gamma\left(p_{0}\right)$ then $\left(0, \alpha_{0}\right)$ is a subcritical (unstable) bifurcation point of (4.3), and if $\gamma<\gamma\left(p_{0}\right)$ then $\left(0, \alpha_{0}\right)$ is a postcritical bifurcation point of (4.3).

Proof. Let $\Phi$ be the key function for (4.3), that is,

$$
\Phi\left(\xi, \alpha, \beta_{0}\right)=-E\left(\tilde{x}\left(\xi, \alpha, \beta_{0}\right), \alpha, \beta_{0}\right)+\frac{1}{2}\left(\xi-\left\langle\tilde{x}\left(\xi, \alpha, \beta_{0}\right), e\right\rangle\right)^{2},
$$


where $\tilde{x}\left(\cdot, \cdot, \beta_{0}\right)$ is the implicit function of the equation $F\left(x, \alpha, \beta_{0}\right)+(\xi-\langle x, e\rangle) e=0$ such that $\tilde{x}\left(0, \alpha_{0}, \beta_{0}\right)=0$. An easy computation based on (4.5) shows that

$$
\begin{aligned}
\Phi_{\xi \xi \alpha}^{\prime \prime \prime}\left(0, \alpha_{0}, \beta_{0}\right) & =-E_{x x \alpha}^{\prime \prime \prime}\left(0, \alpha_{0}, \beta_{0}\right)(e, e, 1), \\
\Phi_{\xi \xi \xi}^{\prime \prime \prime}\left(0, \alpha_{0}, \beta_{0}\right) & =-E_{x x x}^{\prime \prime \prime}\left(0, \alpha_{0}, \beta_{0}\right)(e, e, e), \\
\Phi_{\xi \xi \xi \xi}^{(4)}\left(0, \alpha_{0}, \beta_{0}\right) & =-E_{x x x x}^{(4)}\left(0, \alpha_{0}, \beta_{0}\right)(e, e, e, e)-3 E_{x x x}^{\prime \prime \prime}\left(0, \alpha_{0}, \beta_{0}\right)(e, e, y),
\end{aligned}
$$

where $y=\tilde{x}_{\xi \xi}^{\prime \prime}\left(0, \alpha_{0}, \beta_{0}\right)$. Let us denote $y=\left(y_{1}, y_{2}\right), y_{i} \in C^{0, \mu}(\bar{D})$ for $i=1,2$. Applying (2.8) we receive

$$
\begin{aligned}
\Phi_{\xi \xi \alpha}^{\prime \prime \prime}\left(0, \alpha_{0}, \beta_{0}\right) & =-2 c_{0}>0, \\
\Phi_{\xi \xi \xi}^{\prime \prime \prime}\left(0, \alpha_{0}, \beta_{0}\right) & =0, \\
\Phi_{\xi \xi \xi \xi}^{(4)}\left(0, \alpha_{0}, \beta_{0}\right) & =\frac{1}{\pi} \iint_{D}\left(6 \gamma e_{1}^{4}-6 \alpha\left(\left(e_{1}\right)_{u}^{2}+\left(e_{1}\right)_{v}^{2}\right)^{2}-3\left(\Delta y_{2}\right)^{2}\right) d u d v .
\end{aligned}
$$

Differentiating the equality $F\left(\tilde{x}\left(\xi, \alpha, \beta_{0}\right), \alpha, \beta_{0}\right)+\left(\xi-\left\langle\tilde{x}\left(\xi, \alpha, \beta_{0}\right), e\right\rangle\right) e=0$ twice with respect to $\xi$ at $\left(0, \alpha_{0}\right)$, we receive

$$
F_{x x}^{\prime \prime}\left(0, p_{0}\right)(e, e)+F_{x}^{\prime}\left(0, p_{0}\right) y-\langle y, e\rangle e=0
$$

and hence

$$
\begin{gathered}
\left\langle F_{x x}^{\prime \prime}\left(0, p_{0}\right)(e, e), e\right\rangle+\left\langle F_{x}^{\prime}\left(0, p_{0}\right) y, e\right\rangle-\langle y, e\rangle=0, \\
E_{x x x}^{\prime \prime \prime}\left(0, p_{0}\right)(e, e, e)+\left\langle F_{x}^{\prime}\left(0, p_{0}\right) e, y\right\rangle-\langle y, e\rangle=0, \\
\langle y, e\rangle=0
\end{gathered}
$$

From this it follows that $F_{x}^{\prime}\left(0, p_{0}\right) y=-F_{x x}^{\prime \prime}\left(0, p_{0}\right)(e, e)$, hence that $F_{x}^{\prime}\left(0, p_{0}\right) y=\left(0,\left[e_{1}, e_{1}\right]\right)$, and finally that $\Delta^{2} y_{2}=-\left[e_{1}, e_{1}\right]$. Consequently, substituting $e_{1}(u, v)=c_{1} J_{0}\left(r \sqrt{-c_{0}}\right)$, we receive

$$
\begin{aligned}
\Phi_{\xi \xi \xi \xi}^{(4)}\left(0, \alpha_{0}, \beta_{0}\right)= & \frac{1}{\pi} \iint_{D}\left(6 \gamma e_{1}^{4}-6 \alpha\left(\left(e_{1}\right)_{u}^{2}+\left(e_{1}\right)_{v}^{2}\right)^{2}\right) d u d v \\
& -\frac{1}{\pi} \iint_{D} 3\left(\Delta^{-1}\left[e_{1}, e_{1}\right]\right)^{2} d u d v \\
= & \frac{c_{1}^{4}}{\pi} \iint_{D}\left(6 \gamma J_{0}^{4}\left(r \sqrt{-c_{0}}\right)-6 \alpha c_{0}^{2}\left(J_{0}^{\prime}\left(r \sqrt{-c_{0}}\right)\right)^{4}\right) d u d v \\
& +\frac{c_{1}^{4}}{\pi} \iint_{D} 12 c_{0}^{3}\left(\Delta^{-1}\left(\frac{1}{r} J_{0}^{\prime \prime}\left(r \sqrt{-c_{0}}\right) J_{0}^{\prime}\left(r \sqrt{-c_{0}}\right)\right)\right)^{2} d u d v \\
= & 12 \gamma c_{1}^{4} \int_{0}^{1} r J_{0}^{4}\left(r \sqrt{-c_{0}}\right) d r-12 \alpha c_{1}^{4} c_{0}^{2} \int_{0}^{1} r\left(J_{0}^{\prime}\left(r \sqrt{-c_{0}}\right)\right)^{4} d r \\
& +24 c_{1}^{4} c_{0}^{3} \int_{0}^{1} r\left(\Delta^{-1}\left(\frac{1}{r} J_{0}^{\prime \prime}\left(r \sqrt{-c_{0}}\right) J_{0}^{\prime}\left(r \sqrt{-c_{0}}\right)\right)\right)^{2} d r .
\end{aligned}
$$

Let $D=-6 \Phi_{\xi \xi \xi \xi}^{(4)}\left(0, \alpha_{0}, \beta_{0}\right) / \Phi_{\xi \xi \alpha}^{\prime \prime \prime}\left(0, \alpha_{0}, \beta_{0}\right)$. Then $D>0$ if and only if $\gamma<\gamma\left(p_{0}\right)$, and $D<0$ if and only if $\gamma>\gamma\left(p_{0}\right)$. By Theorem 3.3, the proof is finished. 
Consequently, we receive the following theorem.

Theorem 4.2. If $\gamma \neq \gamma\left(p_{0}\right)$ then $\left(0, p_{0}\right)$ is a bifurcation point of (2.3). Moreover, if $\gamma>\gamma\left(p_{0}\right)$ then $\left(0, p_{0}\right)$ is a subcritical (unstable) bifurcation point with respect to $\alpha$, and if $\gamma<\gamma\left(p_{0}\right)$ then $\left(0, p_{0}\right)$ is a postcritical bifurcation point with respect to $\alpha$.

\section{Acknowledgment}

The first author was supported by Grant KBN no. 2 P03A 04522 and the second author was supported by Grant KBN no. 1 P03A 04229.

\section{References}

[1] L. Bauer and E. L. Reiss, Nonlinear buckling of rectangular plates, J. Soc. Indust. Appl. Math. 13 (1965), no. 3, 603-626.

[2] M. S. Berger, On von Kármán's equations and the buckling of a thin elastic plate. I. The clamped plate, Comm. Pure Appl. Math. 20 (1967), 687-719.

[3] A. Yu. Borisovich, Functional-topological properties of the Plateau operator and applications to the study of bifurcations in problems of geometry and hydrodynamics, Minimal Surfaces, Adv. Soviet Math., vol. 15, American Mathematical Society, Rhode Island, 1993, pp. 287-330.

[4] A. Yu. Borisovich, Yu. Morozov, and Cz. Szymczak, Bifurcation of the forms of equilibrium of nonlinear elastic beam lying on the elastic base, preprint, 2000.

[5] C.-S. Chien and M.-S. Chen, Multiple bifurcation in the von Kármán equations, SIAM J. Sci. Comput. 18 (1997), no. 6, 1737-1766.

[6] K. O. Friedrichs and J. J. Stoker, The non-linear boundary value problem of the buckled plate, Amer. J. Math. 63 (1941), 839-888.

[7] J. Janczewska, Bifurcation in the solution set of the von Kármán equations of an elastic disk lying on an elastic foundation, Ann. Polon. Math. 77 (2001), no. 1, 53-68.

[8] - The study of bifurcation in the von Kármán equations. Applying of topological methods and finite dimensional reductions for operators of Fredholm's type, Ph.D. thesis, Department of Mathematics and Physics, University of Gdańsk, Gdańsk, 2002.

[9] H. B. Keller, J. B. Keller, and E. L. Reiss, Buckled states of circular plates, Quart. Appl. Math. 20 (1962), 55-65.

[10] Yu. Morozov, The study of the nonlinear model which describes the equilibrium forms, fundamental frequencies and modes of oscillations of a finite beam on an elastic foundation, Ph.D. thesis, Department of Applied Mathematics, University of Voronezh, Voronezh, 1998 (Russian).

[11] J. H. Wolkowisky, Existence of buckled states of circular plates, Comm. Pure Appl. Math. 20 (1967), 549-560.

Andrei Borisovich: Department of Mathematics, University of Gdańsk, ul. Wita Stwosza 57, 80952 Gdańsk-Oliwa, Poland

E-mail address: andbor@math.univ.gda.pl

Joanna Janczewska: Department of Technical Physics and Applied Mathematics, Gdańsk University of Technology, ul. Gabriela Narutowicza 11/12, 80-952 Gdańsk, Poland

E-mail address: janczewska@mifgate.pg.gda.pl 


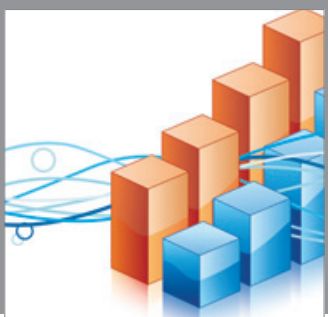

Advances in

Operations Research

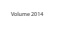

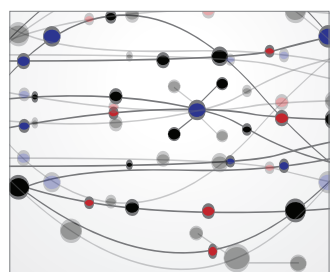

\section{The Scientific} World Journal
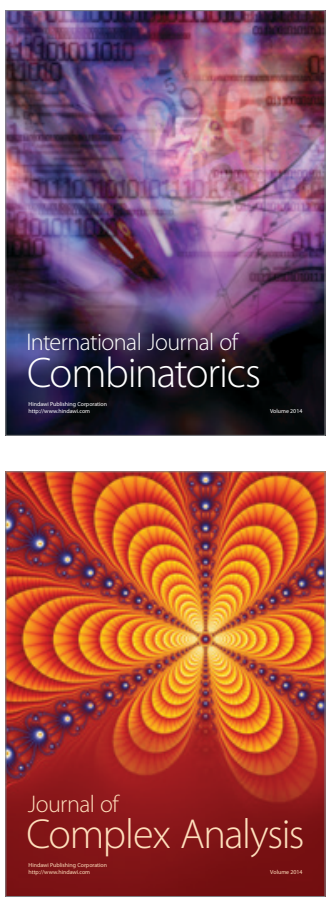

International Journal of

Mathematics and

Mathematical

Sciences
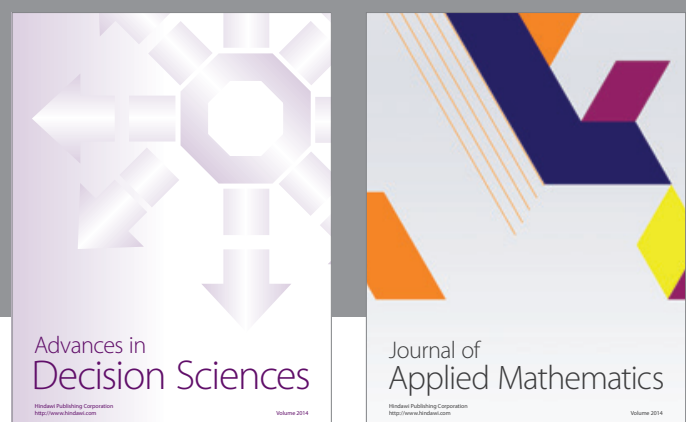

Journal of

Applied Mathematics
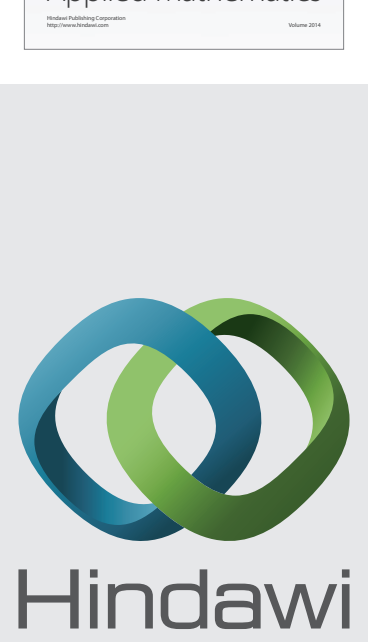

Submit your manuscripts at http://www.hindawi.com
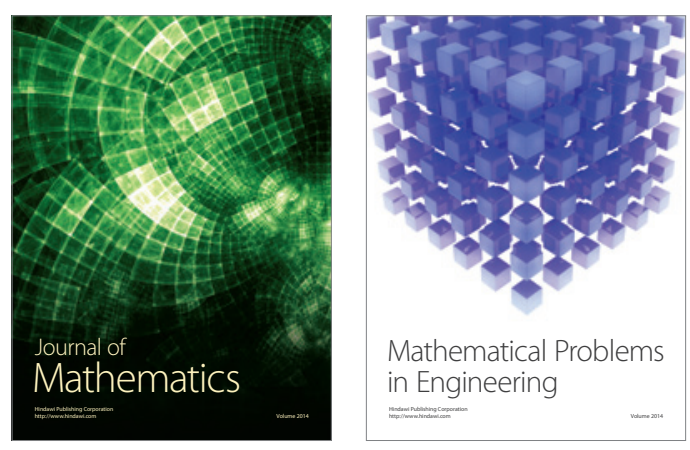

Mathematical Problems in Engineering
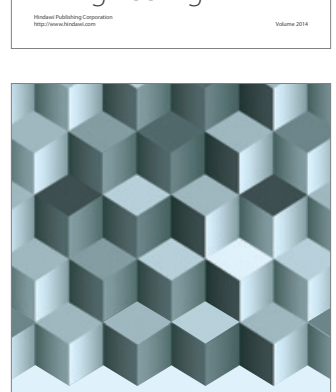

Journal of

Function Spaces
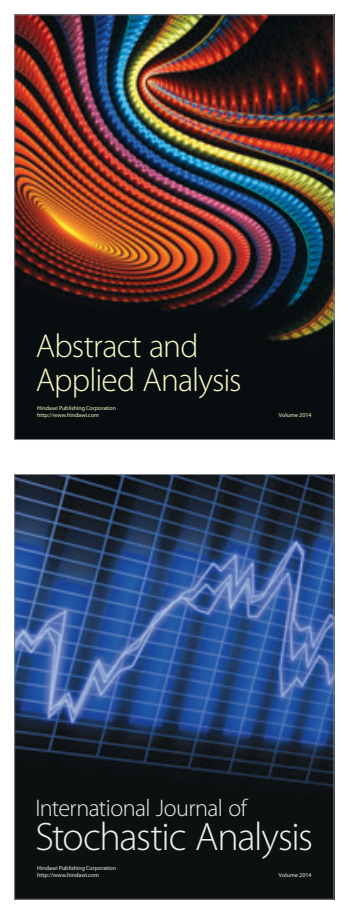

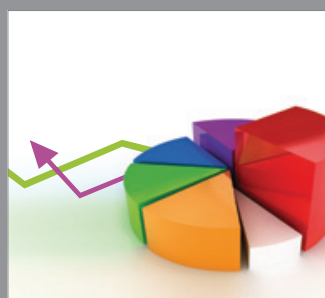

ournal of

Probability and Statistics

Promensencen
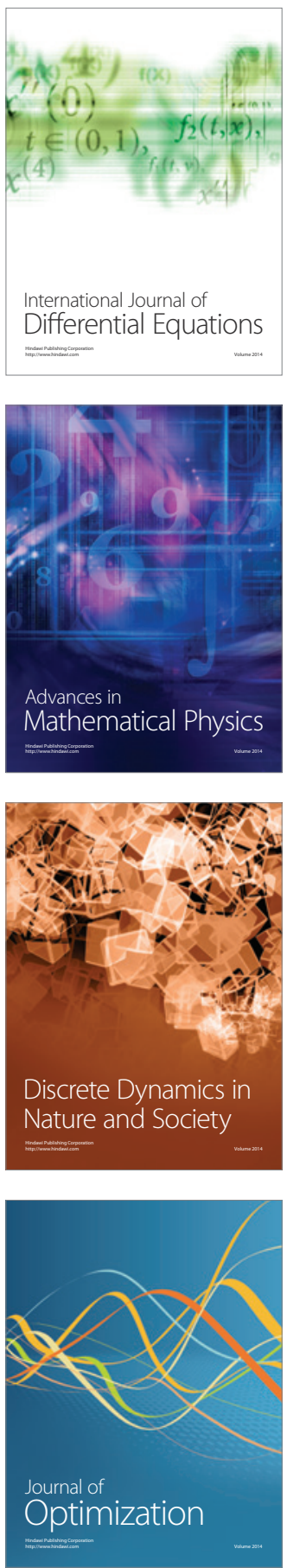